Magnitude Museum: Game-based Learning for Nanosizes, Dimensions, and Nanotechnology Terminology

Dr. Reza Kamali-Sarvestani, Utah Valley University

Brian Durney, Utah Valley University

Brian Durney teaches computer science at Utah Valley University. His research interests are educational games and game AI. 


\title{
Magnitude Museum: Game-Based Learning for Nanosizes, Dimensions, and Nanotechnology Terminology
}

\begin{abstract}
$\underline{\text { Abstract }}$
Magnitude Museum is an educational game that helps students develop a sense of scale and understand the terminology of nanotechnology. Educational and entertainment aspects of the game complement and reinforce each other. Story elements strengthen the learning value of the game and make it more fun. In Magnitude Museum, students explore a 3D virtual museum where each floor of the museum represents an order of magnitude of size in meters. Each floor contains exhibits of objects for that order of magnitude. The top floor (10 to the 26th power) has an exhibit that represents the entire universe and the lowest floors (10 to the minus 15th) have exhibits about subatomic particles. Puzzles in the game help students develop their abilities in estimating size in orders of magnitude, converting units, and using and defining nanotechnology terminology. The puzzles are generated dynamically and range from simple drill-style puzzles to more complicated story-based puzzles.
\end{abstract}

\section{$\underline{\text { Introduction }}$}

What is a scanning electron microscope? How does a transistor compare to a virus in terms of size? How can meters be converted to feet, or feet to meters? Magnitude Museum is an educational game that helps students answer questions like these as they learn the terminology and applications of nanotechnology, develop a sense of scale, and understand units of size.

In the game, each floor of the museum represents an order of magnitude of size in meters, with the exhibits on a floor displaying information about things that are in that size range. For example, on the floor representing 10 to the second power, a player will find exhibits for things whose size is in the range of 100 meters to 999 meters, such as a skyscraper or a cruise ship. Some of the exhibits display information about things that are related to nanotechnology, like a scanning electron microscope or a buckyball.

A player can move a game character from one floor of the museum to another using an elevator that has floor buttons labeled with numbers, with the button for the top floor labeled 26 (for 10 to the 26th power) and the bottom floor button labeled -10 (for 10 to the minus 10th power). Some floors don't have any exhibits, but still have buttons in the elevator.

As the elevator moves from floor to floor, it displays an image of an exhibit for each floor that it passes. In that way, the elevator works something like an interactive version of the "Powers of Ten" video [1] and helps players understand powers of ten and exponents.

During the course of the game, players explore the museum and solve puzzles. Some puzzles require the player to estimate the size of an object by finding the floor with an exhibit of that object and then clicking on that exhibit. Other puzzles give the player a definition or description of an object and then require the player to click on the exhibit that matches the description. 
Critics of educational games sometimes compare them to chocolate-covered broccoli and say that instead of making learning more fun they actually make it worse, just as chocolate-covered broccoli is less appetizing than plain broccoli [2]. In this paper we describe how Magnitude Museum was designed to avoid that problem and provide effective learning and fun.

Our approach was to begin with the pedagogical design of the game because our primary goal was to create software that helps students learn. To guide and evaluate the pedagogical design we applied five learning principles to teaching nanotechnology concepts. Those learning principles and their application are explained in the next section.

After completing the pedagogical design we looked at ways to make the game fun. In this part of the design we used a taxonomy of eight kinds of game fun to develop game mechanics. Because those eight kinds of game fun include a wide range of possibilities we were able to add gameplay elements that made the game fun without diminishing its educational value. The third section of this paper describes how we used the eight kinds of game fun and discusses the relationship between the learning principles and the kinds of game fun. An important part of that discussion is the conclusion that the learning principles and the kinds of game fun are not incompatible and can complement and reinforce each other.

One of the eight kinds of fun is narrative or drama. We consider this an especially important concept because not only are stories and story elements used to make games fun, they also have great educational value. In the fourth section of this paper we show how story elements contribute to Magnitude Museum's educational value and make the game more fun.

After briefly describing our implementation of the game, we conclude by summarizing our approach to using the five learning principles and the eight elements of fun to make games that are educational and fun.

\section{$\underline{\text { Five learning principles }}$}

We have found that five learning principles described by Durney and Harris [3], [4] are valuable concepts in designing educational materials. In this section we explain those principles and how we used them in the pedagogical design of Magnitude Museum.

The five learning principles are:

-People learn what they practice, and only what they practice.

- Practice conditions should be as close to usage conditions as possible.

-Feedback is necessary for learning to occur.

-Effort is proportional to confidence and perception of usefulness.

- Learners need concrete instances.

\section{People learn what they practice, and only what they practice.}

The first learning principle we consider is that people learn what they practice, and only what they practice. A person can't learn how to ride a bicycle by watching someone else do it or by reading a book, nor can a student learn how to program by listening to a lecture or watching a 
video. They learn to do those things by practicing. In order for Magnitude Museum to help players learn about nanotechnology, it needs to provide opportunities for them to practice.

Application of this learning principle required us to decide what things we wanted students to learn from the game and then make sure that they practiced those things while playing the game. The general learning goals for Magnitude Museum are for students to learn the terminology and applications of nanotechnology, develop a sense of scale, and understand units of size. From those general learning goals, we derived the following measurable learning objectives.

Students should be able to:

-Estimate the size of various objects in orders of magnitude

- Convert units of size

- Recall definitions of nanotechnology terms

- State examples of nanotechnology applications

In Magnitude Museum, players practice doing these things by solving puzzles that are dynamically generated by the game. There are four types of puzzles:

Estimating sizes: This type of puzzle identifies an object, such as a postage stamp or a virus, and asks the player to find that object in the museum. Since each floor of the museum represents a certain order of magnitude of size, finding the object requires the player to correctly estimate the size of the object in an order of magnitude.

Converting units of size: To practice this skill, players are given two or more objects and asked to compare the magnitudes of their sizes with different units. For example, the puzzle might ask which is greater, the height of the Golden Gate Bridge in miles or the length of a mouse in feet. To solve this puzzle, a player can find the exhibits for those two objects, learn their sizes in meters, convert the sizes to the given units, and compare the magnitudes. (Note that all exhibits state the sizes of objects in metric units. Puzzles requiring conversion to non-metric units are used only with American students who might be less familiar with metric units.)

Recalling definitions of nanotechnology terms: For this kind of puzzle, players are given a definition of a nanotechnology term, like "scanning electron microscope" and asked to find the exhibit that depicts the term. In some cases, players might need to find characters in the game to solve the puzzle. For instance, the puzzle might say to find a person who uses nanoscale lithography in their job.

State examples of nanotechnology applications: To help players learn about applications of nanotechnology, players are asked to find game characters who have benefited from various kinds of nanotechnology applications, including things like electronic devices, solar cells, and sporting goods.

J. P. Gee notes the importance of practice and states that games provide a good context for practice. He writes: 
There is a paradox involving skills: People don't like practicing skills out of context over and over again, since they find such skill practice meaningless, but, without lots of skill practice, they cannot really get any good at what they are trying to learn. People learn and practice skills best when they see a set of related skills as a strategy to accomplish goals they want to accomplish. [5]

An educational game can encourage practice by providing a context where skills are part of strategies used to accomplish goals in the game.

One potential problem for educational games is that the ratio of practice time to total time could be small. When players are doing things in the game that are not related to practicing (solving puzzles in this case) they are not learning, so we designed the game to minimize the time that players spend on other activities.

\section{Practice conditions should be as close to usage conditions as possible.}

The second learning principle states that a student will be able to transfer learned abilities from school to work if school conditions are as close to work conditions as possible. At first glance, this learning principle seems to be a difficult principle to apply to an educational game because games are not jobs, or like jobs. In fact, the more a game is like a job, the less fun it is likely to be, resulting in the chocolate-covered broccoli syndrome.

We can resolve this issue by looking at the usefulness of models. By definition, a model is not the thing that it represents: a model airplane is not an airplane, and a statistical model of a wolf population is not the same thing as an actual wolf population. However, a model is like the thing it represents in certain important ways, which makes the model useful for doing things that can't be done with the real thing.

What this means for educational games is that we must determine the ways in which the game (or model) conditions must be like the real usage conditions for learning to be effective and make sure that those conditions are as close to the real conditions as possible. Conditions that are less important can be changed to make the game more fun.

Because Magnitude Museum is an introductory game the real-life usage conditions are not well defined. Students will need to estimate sizes, convert units of size, and use nanotechnology terms in a variety of jobs and nanotechnology applications, and there is no single description of usage conditions that covers all of those situations. However, this learning principle is still important in the pedagogical design of the game. Although some parts of the game have whimsical or fantastic aspects, the puzzles do not. That means that when the students are practicing they are focused on real-life objects and concepts.

\section{Feedback is necessary for learning to occur.}

This principle states that learners need feedback to learn. One advantage of educational games like Magnitude Museum is that students don't have to wait for feedback like they often do for tests or homework that is turned in to a teacher for grading. It's also true that a program can't give 
the same kind of feedback that a teacher or grader can, but for the kinds of problems students are solving in Magnitude Museum a program can give good feedback and the student doesn't have to wait.

\section{Effort is proportional to confidence and perception of usefulness.}

Durney and Harris [4] explain that effort can be considered to be the product of confidence and perception of usefulness. If either one is zero, then the student will make no effort to learn. To help build student confidence, the puzzles in the first phase of Magnitude Museum are relatively easy and there isn't much time pressure in that phase of the game. Players have time to explore the museum and learn from the exhibits and puzzles before they go on to later phases of the game where puzzles become more difficult.

Players learn about the usefulness of nanotechnology by interacting with non-player characters (NPCs). Some NPCs tell players about their jobs in nanotechnology, so that players learn about careers in nanotechnology. Other NPCs are looking for ways to improve products, like tennis racquets or phones, and give players the opportunity to learn about how advances in nanotechnology have improved the things that they buy and use.

\section{Learners need concrete instances.}

The fifth learning principle states that learners must form their own concepts and cannot receive them directly from a teacher. Learners form concepts by generalizing from examples. Teachers can help learners by providing examples, but a teacher cannot directly transmit a concept to a learner.

For example, a teacher might tell a learner the concept "round things roll" but the student won't understand without examples. By providing two examples, such as a tennis ball and a pencil, the teacher can help the learner understand the concept. In explaining the concept of recursion, a teacher can give two examples of recursive functions, perhaps with one function that's tail recursive and one that isn't.

Gee describes a similar idea when he states that people usually think and reason in terms of examples and experiences rather than generalities:

Humans do not usually think through general definitions and logical principles. Rather, they think through experiences they have had and imaginative reconstructions of experience. You don't think and reason about weddings on the basis of generalities, but in terms of the weddings you have been to and heard about and imaginative reconstructions of them. It's your experiences that give weddings and the word 'wedding' meaning(s). Furthermore, for humans, words and concepts have their deepest meanings when they are clearly tied to perception and action in the world. [5]

In Magnitude Museum, players learn nanotechnology concepts and concepts relating to dimensions and scale. The exhibits and characters in the game provide examples of those concepts. Some exhibits are things that players can see and are already familiar with: cities, 
mountains, soda cans, coins, and so on. Other things are things that people can't directly see, but are things that players have heard about, such as pollen, viruses, and atoms. By seeing how unfamiliar nanotechnology objects, like nanotubes and buckyballs, relate to familiar objects, like atoms and viruses, students can better form concepts about the sizes of those nanotechnology objects.

\section{Eight kinds of fun}

What makes a game fun? Answers to that question are many and varied. We find that the game taxonomy defined by Marc LeBlanc [6] and discussed in detail by Greg Costikyan [7] is a good way to analyze the fun factor in games. The eight pleasures they define are sensation, fantasy, narrative, challenge, fellowship, discovery, expression, and submission. We examine each of the eight game pleasures and look at the relationships of those eight pleasures to the learning principles that were previously described.

\section{Sensation}

The first game pleasure is sensation, or sensory pleasure. It is possible to make a fun game that has little or no sensory pleasure, as Costikyan notes [7], but sensory pleasure can enhance a game. This kind of game fun is significant for educational games because sensory elements such as images, animation, and music can be added to the game without affecting the correspondence between practice conditions and real usage conditions.

\section{Fantasy}

In this context, fantasy refers to being immersed in a different environment, or losing yourself in the game's setting. LeBlanc et al use the term "make believe" [6] and Costikyan uses the terms "sense of place" and "immersion" [7]. Obviously, Magnitude Museum can't be a complete fantasy world and still have educational value. However, it can have fantasy elements that are orthogonal to the real-world educational aspects of the game.

One important issue for a sense of immersion is that the game should be self-contained. If players are required to do research on the Internet or read books or papers that are not part of the game then they won't be immersed. Not all games have to, or try to, provide a sense of immersion, but Magnitude Museum is designed to be an immersive game and thus is selfcontained.

\section{Narrative}

The narrative game pleasure isn't necessarily about a story in the game. LeBlanc et al use the phrase "game as drama" [6], and Costikyan summarizes it as "rising tension, leading to a climax and a sense of accomplishment" [7]. The only way that a typical homework assignment could be thought of as having rising tension is that such assignments usually have easier problems at the beginning and more difficult problems near the end. 
We have incorporated this game aesthetic into Magnitude Museum by adding story elements to the game. The player takes on the role of a student at the Magnitude Academy who is on a team that is trying to win the coveted Nanotech Cup. During the first phase of the game, the character is preparing for the tournament but the stakes are relatively low and there is no pressure. The second phase of the game is the qualifying round of the tournament and the character is competing against other students (who are computer-controlled characters) so there is narrative tension and time pressure. The third phase of the game is the final round of the tournament and is the most exciting because the Nanotech Cup will be awarded to the winner.

Adding this storyline makes the game more interesting and fun because of the narrative tension, but it does not change the amount of time that the player spends practicing the things that the game teaches: estimating sizes, converting units, and nanotechnology terminology and applications.

We believe that stories and story elements, such as characters and narrative tension, have great value in games, educational or otherwise, and in education in general. In the next section of this paper we examine the role of stories and story elements in Magnitude Museum in more detail.

\section{Challenge}

Costikyan [7] defines struggle, or opposition, as an essential element of games, and challenges provide that opposition to the player. It is important for the player to understand how the challenge relates to the game, and the player must have the resources and abilities to overcome the challenge. Likewise, students doing a traditional homework assignment must understand the usefulness of the thing they are practicing and have confidence that they can do it.

If the challenges in a game are too difficult, the player might have little confidence, which is a problem from the point of view of Learning Principle 4, which states that effort is proportional to confidence and perception of usefulness. However, making challenges too difficult is a problem for any game, educational or not. Good game design practices dictate that game challenges should be carefully tuned and, where possible, adjusted for different kinds of players.

\section{Discovery}

Like challenge, discovery is an important part of both games and learning. Educational games can have two kinds of discovery: discovery of the game world and how it works and discovery of the real-life concepts that the game teaches. For Magnitude Museum, we made the decision that those two kinds of discovery should be as closely related as possible.

There is a trade-off between discovery, narrative tension, and challenge. In order to provide a sense of drama there must be rising tension, which means the player is increasingly challenged and facing time pressure. Those things can make the game exciting and dramatic, but they don't allow the player opportunities to explore, discover, and learn.

To address that issue, Magnitude Museum has three phases. During the first phase, the player has puzzles to solve, but they are more open-ended and there is little time pressure so he or she has 
opportunities to explore and learn about nanotechnology. The second phase is a qualifying round with competition from rival NPCs so there is more at stake from the game point of view. Tension increases and there are fewer opportunities to explore, but there will still be some discoveries. The final phase, which is the final round of the competition, is a chance for the player to show what he or she has learned rather than an opportunity for further discovery.

Discovery is an area where educational topics can contribute to entertainment aspects of a game. Creating an interesting and complex game setting is an expensive undertaking and even when a lot of time and resources are expended the game world might appear to be shallow and uninteresting. For educational topics, a complex body of knowledge already exists, and with good use of game design concepts and skills can be the base of an entertaining game.

\section{Fellowship}

Fellowship includes the social elements of a game. MMORPGs (Massively Multiplayer Online Role Playing Games) allow many opportunities for social interaction, and there are many other kinds of multiplayer games that also allow social interaction. The current version of Magnitude Museum is not a multiplayer game, so fellowship is not a core aesthetic. We are considering adding multiplayer functionality in a later version of the game.

Costikyan writes about fellowship in terms of a sense of community. That means that even games that are not multiplayer games can provide fellowship if the game has a community, and that community can be developed by interaction outside of the game. In the case of Magnitude Museum, a web site could list names (actual names or in-game character names) of people who qualified for the final tournament round and people who won the final tournament.

\section{Expression}

Games that are strong in the area of expression allow players to customize various aspects of the game. For example, role-playing games usually allow players to create a character and choose the character's name, attributes (such as strength or intelligence), and skills. In MMORPGs players can also customize the appearance of their character. Sandbox games, like Minecraft, allow even more expression and allow players to change the world.

Expression doesn't conflict with the learning principles described earlier in this paper, or with educational aspects of games in general, but it is not a core aesthetic for the current version of Magnitude Museum.

\section{Submission}

LeBlanc et al use the phrase "game as pastime" [6], which we interpret as referring to games that require little thought or effort. As such, this is the aesthetic that is least relevant for Magnitude Museum and is not a core aesthetic.

Of the eight game aesthetics or game pleasures, the ones that are most relevant for Magnitude Museum are discovery, challenge, narrative, fantasy, and sensation. 
After examining the relationships of the eight game pleasures to the five learning principles, we have found there is no inherent contradiction between the learning principles and the game pleasures, and therefore there is no reason why educational games cannot be fun.

\section{$\underline{\text { Stories }}$}

Stories can be used in games in two main ways: as scripted stories or as emergent stories. Scripted stories are written in advance. They might include some choice points where the player's decisions influence the outcome of the story but have limited flexibility. The more choice points that are added, the less likely it is that the story will be a coherent, engaging story. Games that use scripted stories often have low replay value because the more the player knows about the story and how it ends, the less suspense and narrative tension there will be.

Because of the limitations and disadvantages of scripted stories, some game designers use the idea of emergent stories in their games. Rather than writing a story in advance, they add story elements to the game, such as interesting characters and obstacles that those characters encounter as they attempt to reach their goals.

In Magnitude Museum, we used the emergent story approach as a way of making the game more fun and better for learning while maximizing replayability. Since emergent stories are not scripted, designers can't directly control emergent stories, but designers can add elements to the game to support and promote emergent stories. We focused on two kinds of story elements: characters and narrative tension.

\section{Characters}

In some games the player's character has a backstory and detailed attributes, but in Magnitude Museum the player's character is left deliberately vague so that the player can fill in whatever story or attributes he or she wants. The only backstory is that the player's character is a student in the Nanotech Academy and is competing for the Nanotech Cup.

Instead of focusing on the player's character, we emphasize non-player characters (NPCs), including rival students who are also competing for the Nanotech Cup, mischievous magpies who steal from the player's character but will sometimes help if the player can give them something they want, and quirky robots that have limited communication but can sometimes help the player. The player can also interact with museum patrons and employees.

By adding these characters to the game, we make the game more fun without decreasing the learning value of the game. In fact, we postulate that adding story elements to the game increases its learning value. In his book, Story Smart [8], storyteller Kendall Haven argues that stories are a valuable way to help listeners understand, remember, and apply information.

It's important to note that Haven is talking about scripted stories told by a person, not about emergent stories in games. However, Haven writes that listeners think in terms of stories and often infer connections, motive, and intent to fill in missing story elements. Our hypothesis is 
that if a game provides important story elements, players will create a story in their minds that will make the game more fun and engaging.

\section{Drama}

One of the eight game pleasures that Costikyan and LeBlanc et al discuss is narrative tension or drama. Costikyan describes drama in games as: "rising tension" [7]. Haven also writes about tension and drama when he talks about risk and danger being essential story elements [8].

What makes a game dramatic? Haven states that risk and danger "create excitement, tension, drama". He then defines risk as "the probability of failure". If there is little chance of failure there is no drama or excitement.

There is an apparent conflict between the story element and game pleasure of drama and the learning principle that says students won't be motivated if they are not confident. However, that conflict can be resolved by recognizing that tension doesn't have to be, and shouldn't be, high from the beginning of the game to the end. Instead, tension should rise as the game progresses, with occasional small drop-offs and occasional peaks. Costikyan describes it as "lots of little peaks of tension, with moments to catch your breath between" [7]. Along the same lines, the designers of the game Left 4 Dead designed an AI system to estimate the players' level of "emotional intensity" (or tension) and adjust game events to create maximal excitement and drama [9].

The need for rising tension, not continuously high tension, provides a way to reconcile confidence and drama. In early parts of the game, tension is relatively low and the player has an opportunity to develop confidence. There is a risk of failure, but because of the risk of failure the sense of accomplishment from solving a puzzle will be greater, which in turn will inspire greater confidence.

The three phases of Magnitude Museum (training, qualifying tournament round, and final tournament round) are progressively more dramatic and exciting because there is more at stake in each round than in the proceeding one. Within each phase there is also rising tension, with occasional peaks and drop-offs. This approach works well from the learning point of view because it allows the player's confidence to grow, and it works well from the point of view of fun and emergent stories.

The game adjusts tension by controlling several parameters for puzzles. Perhaps the most basic, but most important, parameter is putting time limits on puzzles. That leads to rising tension because when there appears to be plenty of time to solve the puzzle there isn't much tension, but tension increases as time decreases.

Another important way to manage tension is by adjusting the difficulty of puzzles. The more difficult a puzzle is, the greater the risk of failure, and greater risk means greater tension. In Magnitude Museum, puzzles about familiar objects and concepts, like cars, football fields, and ants are less difficult than puzzles about less familiar objects and concepts, like a buckyball or a scanning electron microscope. Complexity of puzzles also plays a role in tension levels. More 
complex puzzles, like unit conversion puzzles or multipart puzzles, contribute more to tension than simple puzzles.

Perhaps the most interesting factor in drama is competition. In the second (qualifying tournament round) and third (final tournament round), the player's character competes against non-player characters. Competition is interesting because it contributes to a sense of story in two ways: in terms of drama and in terms of characterization. Drama and tension can be managed by adjusting the strength of competition, with stronger competition causing greater tension.

Competition strengthens characterization if players benefit from analyzing the strengths and weaknesses of their rivals. If those strengths and weaknesses relate to the educational topic of the game (in this case, nanotechnology and sense of scale) then the learning value of the game is also enhanced. For example, a certain non-player character might be good at sizes that are positive orders of magnitude, but not good at very small sizes. The player can then adjust his or her strategy accordingly, perhaps by choosing puzzles that are on the lower floors of the museum where there will be less competition. In that way the player will be thinking about game topics and enjoying the game at the same time that he or she is thinking about educational topics and learning, and the entertainment and educational aspects of the game will complement and reinforce each other.

\section{$\underline{\text { Implementation }}$}

We are using the Unity ${ }^{\mathrm{TM}}$ game engine (unity.com) and the C\# programming language to implement Magnitude Museum with 3D graphics. We are also implementing a 2D browserbased version with JavaScript, HTML5, and CSS. Both the 3D version and the $2 \mathrm{D}$ browser version are currently playable and include the elevator, basic puzzles, and some important game mechanics. More complex puzzles involving characters and story elements have not yet been implemented.

\section{$\underline{\text { Summary }}$}

In this section we summarize our approach to designing Magnitude Museum and briefly discuss our future work. Our approach applies the five learning principles described by Durney and Harris [3], [4], and the eight game pleasures stated by LeBlanc et al [6] and discussed in more detail by Costikyan [7].

As with most design activities, this is an iterative process, but we will describe it as a five-step sequential process.

\section{Determine learning objectives, practice activities, and core game mechanics}

Our first step was to write measurable learning objectives that stated what we wanted students to be able to do after playing the game. We then decided how students could practice doing those things and reach the learning objectives. We then based the core game mechanics on those practice activities so that students can spend as much time as possible doing those activities. This step relates to Learning Principle 1, which states that students learn only what they practice. 
Our learning objectives for Magnitude Museum specified that students should be able to estimate sizes, convert units of size, recall definitions of nanotechnology terms, and state examples of nanotechnology applications. The core game mechanic that is based on these objectives is solving puzzles, with one type of puzzle for each objective. Other, related game mechanics, are moving from floor to floor of the museum and viewing and interacting with exhibits.

\section{Design the game's setting so that practice can be done and feedback can be given without breaking immersion.}

This step relates to the idea that a sense of fantasy or immersion help make games fun. With that in mind we designed the museum to be an immersive environment with a whimsical feel. However, this step also relates to Learning Principle 2, which says that practice conditions should be as close to usage conditions as possible, so we made sure that the whimsical and fantastic elements did not interfere with practice activities.

We also made sure that practice and feedback are closely integrated into the game's setting and mechanics so that players will feel immersed in the game even when, or especially when, practicing the learning objectives. If the fun parts of the game are unrelated to the learning parts of the game, then the game will come across as chocolate-covered broccoli and will not be fun or educational.

\section{Add story elements}

With the practice activities, core game mechanics, and setting of the game established, we proceeded to add story elements to the design. We focused on two important story elements: characters and narrative tension. These story elements are important for learning and for making the game fun.

The player's character is a student at Nanotech Academy and is competing to win the Nanotech Cup. Other characters in the game include students who are competing with the player's character, mischievous magpies, robots, and museum patrons and employees. As the player's character interacts with these characters, emergent or unscripted stories develop that make the game more engaging. Each character has distinctive characteristics and each is related to the core ideas of the game, which are nanotechnology, scale, and units of size.

To add excitement and drama to the game we made the Nanotech Cup tournament with three phases: a training phase, a qualifying round, and the final tournament round. The training phase is relatively slow paced and gives the player time to explore. There is more pressure in the second phase where only a limited number of characters can qualify for the final round, and the third phase has the most drama and tension because only one character can win the cup. Within

each phase there are small peaks and drop-offs, depending on the difficulty and complexities of the puzzles.

This step relates to the narrative game pleasure but can also relate to discovery and immersion. Learning Principle 4 is also relevant to this step. That principle relates effort to confidence and 
perception of usefulness. If tension increases too quickly, the player's confidence will decrease and the player will be less motivated.

\section{Add related elements of fun}

In the fourth step we considered each of the eight game pleasures to find ways that we could relate them to the learning topics of the game. For example, for the game pleasure of sensation we looked for interesting and appealing images and sounds for exhibits. We added a video screen to the elevator that shows images from exhibits on floors that the elevator passes by.

For the game pleasure of discovery we included exhibit topics that students are not familiar with so that each floor of the museum provides opportunities for discovery. In this way the learning topics of the game strengthen the game pleasure of discovery.

The game pleasure of challenge is related to learning and practicing, so it is an important one for educational games. We designed puzzles of varying difficulty so players will able to build confidence, which is relevant to Learning Principle 4, but will still be challenged, which is relevant to the game pleasures of challenge and narrative tension.

\section{Add other elements of fun that don't detract from the learning topics}

Finally, we added other elements of fun that aren't directly related to learning but that don't interfere with learning. Adding music to the game isn't related to the learning topics, but it doesn't interfere with them and it increases the sensation game pleasure. Likewise some of the more whimsical and fantastic elements of the game (such as talking magpies and some visual aspects of the museum rooms) add to a sense of fantasy and sensation.

We haven't yet incorporated the game pleasures of fellowship and expression into the design of Magnitude Museum, but those game pleasures can make the game more fun without interfering with the educational aspects.

\section{Future work}

In the future we plan to make a multiplayer version of Magnitude Museum. That will incorporate the game pleasure of fellowship and will also allow for a greater variety of emergent stories as students compete against and cooperate with other students. Along the same lines, we will allow players to select and customize avatars for their characters in the game, which relates to the game pleasure of expression.

We plan to develop and generalize this approach by applying it to the design of other learning games, in the area of nanotechnology and in other areas. Each educational topic provides different challenges and opportunities, but we believe that the five learning principles and the taxonomy of eight game pleasures provide a helpful foundation for the design of any educational game.

\section{$\underline{\text { Conclusion }}$}


In order to ensure that Magnitude Museum is an effective learning tool for nanotechnology and sense of scale, we have applied five learning principles in its design. In particular, we determined learning objectives for the game and determined what students need to practice to achieve those objectives. We based the core game mechanics on those practice activities so that students get the practice they need as they play the game.

We used a taxonomy of eight game pleasures to find ways to make the game fun as well as educational. We also incorporated story elements into the game as a way of making the game more fun and more educational. We focused on the story elements of characters and narrative tension.

Although some educational games have been criticized for being an unappealing combination of education and entertainment, we have found that with careful design the learning principles and elements of game pleasure can complement and reinforce each other.

\section{$\underline{\text { References }}$}

[1] Eames Office. "Powers of Ten," YouTube, Aug. 26, 2010 [Video file]. Available: https://www.youtube.com/watch?v=0fKBhvDjuy0. [Accessed: March 19, 2018].

[2] E. Smith, "Total Edutainment Forever", Tedium, April 13, 2017. [Online]. Available: https://tedium.co/2017/04/13/edutainment-math-blaster-chocolate-covered-broccoli. [Accessed: March 19, 2018].

[3] C. H. Durney, "Principles of design and analysis of learning systems", in Engineering Education, 63 (6), 406-409, 1973.

[4] C. H. Durney and L. D. Harris, "Using Principles of Learning to Design Learning Systems," in ASEE National Effective Teaching Institute, 1977.

[5] J. P. Gee, "Learning by Design: Good Video Games as Learning Machines". E-Learning, vol. 2, no. 1, March 2005.

[6] R. Hunicke, M. LeBlanc, and R. Zubek, "MDA: A Formal Approach to Game Design and Game Research," in Challenges in Game Artificial Intelligence: Papers from the 2004 AAAI Workshop, 2004.

[7] G. Costikyan, "I Have No Words \& I Must Design: Toward a Critical Vocabulary for Games," in Computer Games and Digital Cultures Conference Proceedings, 2002, vol. 1.

[8] K. Haven, Story Smart: Using the Science of Story to Persuade, Influence, Inspire, and Teach. Libraries Unlimited, 2014.

[9] M. Booth, "The AI Systems of Left 4 Dead," 2009. [Online]. Available: http://www.valvesoftware.com/publications/2009/ai_systems_of_14d_mike_booth.pdf. [Accessed: 05-Feb-2018]. 